\title{
Transitional Care Model: Um Novo Modelo De Gestão De Cuidados Na Comunidade
}

\author{
Transitional Care Model: A New Community Care Management Model
}

\author{
Modelo De Atención Transitoria: Un Nuevo Modelo De Gestión De Atención \\ Comunitaria
}

Tiago Filipe Rodrigues do Nascimento ${ }^{1}$ Maria de Lourdes Gil Patrício Varandas Costa ${ }^{2}$

\section{Resumo:}

As admissões hospitalares têm aumentado, sendo que a taxa de reinternamento hospitalar aumentou aproximadamente $8 \%$. É fundamental adotar medidas que visem diminuir estes valores e consolidem uma transição efetiva entre cuidados de saúde primários e hospitalares. Este artigo tem como objetivo demonstrar uma ferramenta para reduzir os reinternamentos hospitalares dos utentes com fragilidade, em contexto domiciliário, com recurso ao Transitional Care Model (TCM). O trabalho é de natureza quantitativa, descritiva e exploratória, segundo a metodologia do processo de planeamento em saúde, aplicação no domicílio da Edmonton Frail Scale,

\footnotetext{
${ }^{1}$ Enfermeiro. Mestre em Enfermagem na Área de Especialização de Gestão em Enfermagem. Enfermeiro, Santa Casa da Misericórdia de Lisboa. Email: tiago.filipedonascimento@gmail.com ORCID: 0000-0003-3646-9057.
}

Escala de Quedas de Morse e ainda a aplicação de questionário sociodemográfico. A amostra é constituída por 37 utentes, 78,4\% mulheres, $100 \%$ reformados, $67,6 \%$ de classe baixa, 91,8\% tiveram, pelo menos, uma ida ao serviço de urgência hospitalar, 97,3\% apresentam médio a elevado risco de queda, $63 \%$ com fragilidade moderada a severa. Considerando a elevada fragilidade do idoso no domicílio, a aplicação deste modelo permitirá reduzir as barreiras existentes entre os níveis de cuidados, com ganhos em saúde bem como custoefetividade pela redução do número de dias de internamento. Teremos, assim, a prevenção de complicações e melhoria da gestão da doença crônica através da

2 Enfermeira. Enfermeira Especialista em Enfermagem Comunitária, Doutora em Educação. Professora Adjunta, Escola Superior de Enfermagem de Lisboa. Avenida Professor Egas Moniz, 1600-190 Lisboa, Portugal. E-mail: lcosta@esel.pt ORCID: 0000-0003-2681-9375 
aplicação de um novo modelo de gestão de cuidados.

Descritores: Cuidado Transicional; Enfermagem em Saúde Comunitária; Idoso de 80 anos ou mais

\section{Abstract:}

As hospital admissions have increased, the readmission rate has increased by approximately $8 \%$. It is essential to adopt measures aimed at reducing these values and consolidating an effective transition between primary and hospital care. This article aims to demonstrate a tool to reduce hospitalizations of patients with frailty in the home context, using the Transitional Care Model (TCM). The work is quantitative, descriptive and exploratory, according to the method of health planning process, application at home of the Edmonton Frail Scale, Morse Falls Scale and application of sociodemographic questionnaire. The sample is 37 users, $78.4 \%$ of women retired, $67.6 \%$ of lower class, $91.8 \%$ had at least one visit to the emergency room service, $97.3 \%$ of medium and high use. $63 \%$ risk with moderate to severe frailty. In spite of high frailty of the elderly at home, an application of this model that reduces the barriers between levels of care, with health gains as well as economic effectiveness by reducing the number of days of hospitalization. This will prevent complications and improve chronic disease management through the application of a new care management model.

Keywords: $\quad$ Transitional Care; Community Health Nursing; Aged, 80 and over

\section{Resumen:}

A medida que aumentaron los ingresos hospitalarios, la tasa de reingresos aumentó aproximadamente un 8\%. Es esencial adoptar medidas destinadas a reducir estos valores y consolidar una transición efectiva entre la atención primaria y la hospitalaria. Este artículo tiene como objetivo demostrar una herramienta para reducir las hospitalizaciones de pacientes con fragilidad en el contexto del hogar, utilizando el Modelo de Atención Transicional (MTC). El trabajo es cuantitativo, descriptivo y exploratorio, de acuerdo con el método del proceso de planificación de la salud, la aplicación en el hogar de la Escala de Fragilidad de Edmonton, la Escala de Caídas de Morse y la aplicación del cuestionario sociodemográfico. La muestra es de 37 usuarios, el 78,4\% de las mujeres, toda la muestra se ha retirado, el $67,6 \%$ de la clase baja, el 91,8\% tuvo al menos una visita al servicio de urgencias, el 97,3\% 
de uso medio y alto. $63 \%$ de riesgo con fragilidad moderada a severa.

Considerando la existencia de una alta fragilidad de los ancianos en el hogar, una aplicación de este modelo que reduce las barreras entre los niveles de atención, con ganancias de salud y efectividad económica al reducir el número de días de hospitalización. Esto evitará complicaciones y mejorará el manejo de enfermedades crónicas mediante la aplicación de un nuevo modelo de manejo de atención.

Descriptores: Cuidado de Transición; Enfermería en Salud Comunitaria; Anciano de 80 o más Años

\section{Introdução:}

O aumento da esperança média de vida e envelhecimento populacional atual trazem um impacto na sustentabilidade e na gestão dos recursos em saúde (1). Por outro lado, as admissões hospitalares têm vindo a aumentar na realidade europeia e, dessas admissões, muitas dizem respeito a readmissões, sendo que a taxa de reinternamento, em Portugal, sofreu um aumento ao longo da última década, fixando-se em 2018 em valores aproximados de $8 \%$ (2). Este valor situase, mesmo assim, abaixo do valor francês, em que as readmissões hospitalares correspondem a 14\%, um quarto destas evitável (3). Tendo em conta que as taxas de mortalidade são superiores nos episódios de reinternamento (2), surge uma necessidade de reduzir estes valores e de criar uma aproximação estratégica entre a comunidade e os cuidados hospitalares, tendo como foco o utente idoso com uma deficitária gestão do seu projeto de saúde. Metade destes reinternamentos podem também ser explicados pela localização do hospital, condições socioeconômicas, número de profissionais de saúde e a qualidade das estruturas de cuidados de longa duração. Portanto, as taxas de readmissão hospitalar podem ser reduzidas de maneira mais eficaz se forem pensadas estratégias de regresso à comunidade. Fica também patente que há uma necessidade dos profissionais alternarem entre os vários níveis de cuidados de saúde para aumentar a compreensão e consciencialização, eliminando as barreiras existentes, construindo um canal mais próximo de comunicação, assente também num aumento da literacia junto dos clientes e famílias (4). Surge, assim, na literatura, o Transitional Care Model (TCM) como referencial teórico, associado ao modelo de empowerment como garantia da intervenção para a melhoria da gestão de cuidados e, consequentemente, do regime de saúde. Além dos referenciais 
anteriormente descritos, este artigo visa também demonstrar as competências comuns e específicas do Enfermeiro Especialista em Enfermagem Comunitária. Considerando, então, a problemática, este artigo teve como ponto de partida a seguinte questão: a intervenção do Enfermeiro Especialista em EC, com recurso ao TCM, reduz os internamentos/reinternamentos hospitalares? Para dar resposta a esta questão, foi desenvolvida uma pesquisa recorrendo à metodologia scoping review e desenvolvida a respectiva proposta de planeamento em saúde com a finalidade de promover $\mathrm{o}$ empowerment no idoso e família, para reduzir o internamento e reinternamento hospitalar dos utentes numa unidade de saúde. O objetivo deste artigo é relatar os resultados de pesquisa acerca da fragilidade do idoso em contexto domiciliário, permitindo, assim, intervir precocemente para reduzir os reinternamentos, com recurso ao TCM.

\section{Revisão da Literatura:}

O envelhecimento é um percurso inevitável, universal e irreversível, que se inicia antes do nascimento e desenvolve-se ao longo da vida, comprometido por mudanças a nível biológico, psicológico e social $(5,6)$. Prevê-se que 3 em cada 10 residentes, em
Portugal, terão 65 ou mais anos em 2050 (7). Considerando este aumento da esperança média de vida, é de esperar um aumento da prevalência das doenças crônicas e respectivas co-morbilidades, situação que compromete o decurso normal do envelhecimento e que origina internamentos hospitalares por via da descompensação do processo de saúdedoença.

Este envelhecimento encontra-se intimamente ligado a um aumento da fragilidade, que pode ser definida como uma síndrome multidimensional de redução das reservas e redução da resistência a stressores, levando a uma maior vulnerabilidade e, consequentemente, resultados adversos. (8) A fragilidade no idoso resulta de diferentes condições que estão correlacionadas umas com as outras de maneira complexa, destacando-se tanto fatores biológicos ou físicos, como psicossociais. (9) Os internamentos hospitalares e as respectivas readmissões hospitalares têm-se tornado um flagelo, tendo em conta o tempo de internamento, as repercussões destes internamentos bem como os custos inerentes, sejam eles diretos (pelo internamento hospitalar, tratamentos, cirurgias, entre outros) ou indiretos (diminuição da produtividade, aumento da dependência, absentismo laboral por parte do utente e/ou 
cuidadores). O modelo conceitual utilizado foi escolhido tendo por base aquele que pode ser um mecanismo inovador de prestação de cuidados, reduzindo os internamentos hospitalares e promovendo uma transição segura entre os vários níveis de cuidados.

Desta forma importa enquadrar a pertinência das transições que, de acordo com a Teoria das Transições de Afaf Meleis, resultam de mudanças de vida, saúde, relacionamentos e ambiente, implicando uma mudança do cliente e família em cada um destes aspectos, de modo a readaptarem-se a uma nova realidade. (10) Devido à complexidade e diversidade das transições, o enfermeiro deve identificar, clarificar e procurar compreender o processo de transição de um cliente e família e desenvolver intervenções de enfermagem que promovam uma resposta saudável à transição. (10) Esta promoção estará assente no modelo de empowerment, na medida em que este é um processo em que as pessoas e as comunidades adquirem controle sobre as decisões que condicionam a sua saúde. (11) Na realização da pesquisa inicial recorrendo à metodologia scoping review, constatou-se que este modelo já se encontra implementado em vários países, sempre com um denominador comum: o Enfermeiro Especialista em
Enfermagem Comunitária enquanto elemento central do modelo, na perspectiva da sua gestão. É sobre o Enfermeiro que recai a responsabilidade de agregar toda a informação e de garantir a continuidade dos cuidados. A literatura corrobora o fato de que a aplicação do modelo reduz os internamentos hospitalares, bem como respectivas readmissões (12), e é focada a importância de continuar a desenvolver investigação que permita validar a aplicação deste modelo. (13) Vários estudos apontam para o custo efetividade do modelo, especialmente pelos baixos custos dos cuidados prestados na comunidade (14), custos estes que podem ser reduzidos se houver um aumento da literacia em saúde, que tem uma correlação positiva com a redução das readmissões hospitalares. (4)

\section{Metodologia de pesquisa:}

Trata-se de um estudo descritivo e exploratório, de natureza quantitativa, que se caracteriza através da aplicação de instrumentos de coleta de dados, tendo em conta os critérios previstos no TCM: utentes com doenças crônicas, quedas recentes, dependência, saúde mental/cognitiva, internamento nos últimos 30 dias, ou 2 ou mais internamentos nos últimos 6 meses, idade > 80 anos, défice na literacia em 
saúde, barreiras linguísticas, défice no sistema de suporte. Considerando a necessidade de avaliar a fragilidade do idoso, foi utilizado como instrumento de coleta de dados a Edmonton Frail Scale $(15,16)$ e a Escala de Quedas de Morse (17), para permitir a consideração de vários fatores que aumentam a fiabilidade da pesquisa. Adicionalmente foi aplicado um questionário sociodemográfico (sexo, idade, escolaridade, tipo de família, profissão e Escala de Graffar como instrumento de classificação social), com o objetivo de caracterizar a população amostral que será alvo da prestação de cuidados (especializados). A amostra é constituída por 37 utentes em contexto de apoio domiciliário (de uma população de 10209 utentes inscritos na unidade) numa unidade de saúde em Lisboa, numa amostragem por conveniência. Foram respeitadas as dimensões éticas na aplicação dos questionários, garantindo o consentimento dos clientes bem como a confidencialidade dos dados. Os dados foram trabalhados estatisticamente recorrendo ao Statistical Package for Social Sciences $(R)$ 25.0.

\section{Análise e discussão:}

Do questionário sociodemográfico aplicado, obteve-se amostra constituída por 29 mulheres $(78,4 \%)$ e 8 homens
$(21,6 \%)$ com idades superiores a 80 anos, de acordo com critério anteriormente estabelecido, com uma idade máxima de 103 anos e uma média de 88,7 anos. No que respeita ao agregado familiar, a amostra distribuiuse por $21,6 \%(\mathrm{n}=8)$ com família extensa ou alargada, $29,7 \%$ (n=11) com família unitária, 43,2\% ( $\mathrm{n}=16)$ família com dependente e apenas $5,4 \%(\mathrm{n}=2)$ com famílias reconstruídas. Relativamente à escolaridade, $45,9 \% \quad(\mathrm{n}=17)$ não tinha qualquer escolaridade ao que $45,9 \%$ $(\mathrm{n}=17)$ apresentava o ensino básico e apenas $8,1 \%(\mathrm{n}=3)$ o ensino secundário. No que concerne à profissão e tendo em conta a idade e dependência, todos os utentes se encontravam reformados. $\mathrm{Na}$ aplicação da escala de Graffar (18) e tendo em conta a população característica da instituição prestadora de cuidados, 32,4\% ( $\mathrm{n}=12$ ) situam-se na Classe IV - Média Baixa e os restantes 67,6\% ( $\mathrm{n}=25)$ na Classe V - Baixa. Na aplicação da Escala de Morse para avaliação do risco de queda, e tendo em conta que acima do score de 25 pontos existe risco de queda, 97,3\% da amostra $(\mathrm{n}=36)$ apresentava um médio a altorisco de queda. Através da aplicação da Edmonton Frail Scale para avaliação da fragilidade do idoso, uma escala constituída por 9 domínios e 12 itens, com pontuação máxima de 17 pontos, 
obteve-se na variável de cognição (teste do relógio), $37,8 \% \quad(n=14)$ não apresentava qualquer erro, $35,1 \%(\mathrm{n}=13)$ apresentava pequenos erros e $27 \%$ $(n=10)$ apresentava outros erros. No que concerne à variável do estado geral da saúde, 37,8\% ( $\mathrm{n}=14)$ considera ter um excelente/muito bom/bom estado de saúde, 45,9\% (n=17) um estado de saúde razoável e apenas 16,2\% (n=6) um estado de saúde pobre. Ainda na dimensão estado geral, 48,6\% $(n=18)$ da amostra teve mais de 2 entradas no hospital, 43,2\% $(n=16) 1$ ou 2 entradas e apenas $8,1 \%(n=3)$ não teve qualquer entrada no hospital no ano anterior. Relativamente à independência funcional, com as variáveis necessitar de ajuda para preparação de refeições, compras, transportes, utilizar telefone, limpeza, lavandaria, gestão do dinheiro e toma de medicação, em que sim, o utente precisa de ajuda, equivale a um ponto e não, não precisa de ajuda, pontua zero, obtiveram-se os seguintes resultados: $13,5 \%(n=5)$ não precisa de ajuda ou precisa de ajuda para apenas uma atividade, $51,4 \%(\mathrm{n}=19)$ precisa de ajuda para 2 a 4 atividades e $35,1 \%(n=13)$ precisa de ajuda para 5 a 8 atividades. Ao nível do suporte social, ou seja, se quando o utente precisa de ajuda tem alguém que possa estar disponível, 16,2\% $(n=6)$ responderam que sim, têm sempre alguém disponível, 62,2\% $(n=23)$ têm às vezes alguém disponível, e $21,6 \%(n=8)$ nunca têm alguém disponível. Na dimensão da medicação, relativamente à variável de utilizar cinco ou mais medicamentos regularmente por prescrição médica, 29,7\% $\quad(\mathrm{n}=11)$ respondeu que não ao que $70,3 \%(n=26)$ afirmou que toma cinco ou mais medicamentos regularmente. Ainda no domínio da medicação, o item de validar se o utente se esquece de às vezes tomar a medicação, $73 \%$ (n=27) afirma que não se esquece, sendo que os restantes $27 \%$ $(n=9)$ referem que sim, que têm falhas ocasionais. No domínio da nutrição e no item de ter perdido peso e sentido que o vestuário ficou mais largo, 81,1\% (n=30) afirma que não perdeu peso, sendo que $18,9 \%(n=7)$ afirma que sim. No domínio do humor, o item de pergunta que aborda se o utente se tem sentido triste ou deprimido, $45,9 \%(\mathrm{n}=17)$ refere que não e 54,1\% (n=20) assume que sim. $\mathrm{Na}$ avaliação da incontinência, o item refere-se em concreto à dificuldade em manter o controlee da urina, pelo que $27 \%(\mathrm{n}=10)$ responde que não tem qualquer dificuldade a controlar a urina e os restantes $73 \%(\mathrm{n}=27)$ refere que sim, que tem perdas ocasionais ou mesmo incontinência total do esfíncter urinário. No domínio da performance funcional, foi aplicado o teste timed up and go que 
como resultado obteve-se que $24,3 \%$ (n=9 da amostra) demorou 0 a 10 segundos a executar, 43,2\% ( $\mathrm{n}=16)$ demorou 10 a 20 segundos e $32,4 \%$ $(\mathrm{n}=12)$ demorou mais de 20 segundos, não quis ou não conseguiu realizar por estar completamente dependente. Desta forma e de acordo com o somatório das variáveis, obteve-se que $5 \%(\mathrm{n}=2)$ da amostra não apresenta qualquer tipo de fragilidade ( 0 a 4 pontos), $8 \%$ ( $n=3$ ) está aparentemente vulnerável (5-6 pontos), 24\% $(\mathrm{n}=9)$ tem fragilidade leve $(7-8$ pontos), $41 \%(\mathrm{n}=15)$ tem fragilidade moderada ( $9-10$ pontos) e $22 \%$ ( $n=8$ ) da amostra tem fragilidade severa (igual ou superior a 11 pontos).

\section{Discussão:}

De acordo com os resultados obtidos, foi percetível a elevada fragilidade dos utentes da amostra, tendo em conta a sua situação sociodemográfica, de menores recursos financeiros, menor estrutura familiar, menor literacia por escolaridade mais baixa ou ausência desta. Contribui também para a maior fragilidade a idade avançada da amostra que se caracteriza pelo aumento das comorbilidades associadas. Esta maior dependência e vulnerabilidade é compatível com este elevado risco de queda, motivo de um grande número de internamentos e reinternamentos hospitalares, bem como preditor de aumento da dependência do utente. $\mathrm{O}$ valor de $97,3 \%$ de utentes com médio a elevado risco de queda torna premente uma intervenção de promoção de saúde e de capacitação de cuidadores concertada e rápida.

Concomitantemente, na aplicação da Edmonton Frail Scale e na análise dos itens, foi percetível que existe claramente no item "quantas vezes no passado ano deu entrada no hospital", uma vulnerabilidade acrescida e um risco de internamento por agudização ou agravamento da doença crônica. Neste contexto importa monitorizar estas doenças crônicas e permitir uma efetiva prevenção de complicações, orientando e promovendo o empowerment junto dos utentes e cuidadores para que tenham a perceção dos sinais e sintomas associados a desvios do seu padrão de saúde.

Também o domínio do suporte social é motivo de maior preocupação pelo fato de apenas $16,2 \%$ da amostra ter sempre disponível um elemento para dar apoio quando necessário, reforçando a necessidade de um acompanhamento contínuo por parte do Enfermeiro, garantindo, simultaneamente, a acessibilidade rápida em caso de agudização com meios de comunicação céleres e user-friendly como, por 
exemplo, o botão de tele-assistência que pode acompanhar o utente em forma de colar no pescoço.

No que respeita à medicação, são compatíveis os resultados com os achados bibliográficos, na medida em que $70,3 \%$ da amostra se encaixa no conceito de polimedicado, ou seja, com uma toma regular de 5 ou mais comprimidos. Este é também um foco de atenção importante para a necessidade de promoção de uma efetiva reconciliação terapêutica, permitindo uma redução de custos na aquisição desta medicação, mas, acima de tudo, pela redução dos efeitos idiopáticos e das interações medicamentosas decorrentes da toma de um elevado número de medicamentos. Contrariamente ao expectável, $73 \%$ da amostra afirma não ter qualquer esquecimento na toma da medicação, o que demonstra a valorização que os utentes dão ao fato de cumprirem de forma regular a sua toma da medicação e a associação ao seu melhor estado de saúde. No entanto, mantém-se como foco de atenção os remanescentes $27 \%$ da amostra com esquecimentos ocasionais da toma de medicação e consequente agudização da sua doença crônica.

Urge, assim, negociar estratégias para aumento da compliance do regime terapêutico instituído. Ainda no que concerne ao regime terapêutico, o domínio da nutrição pode ter duas interpretações distintas: se por um lado a não existência da perda de peso pode significar a ausência de doença recente, por outro lado pode ser também colocada em causa o regime dietético bem como o exercício físico realizado no contexto domiciliário por parte dos utentes levando a aumento de peso progressivo, não especificado por esta escala mas que será importante valorizar.

Considerando os resultados apresentados, surgiram como diagnósticos comunitários potenciais (de acordo com a Classificação Internacional para a Prática de Enfermagem): crise de saúde aguda, risco de queda, problema de continuidade de cuidados, falta de suporte social, mobilidade comprometida e défice de autocuidado. Assim, e dando continuidade à metodologia de planeamento em saúde, procedeu-se à priorização dos diagnósticos de acordo com o método do plano de Biscaia tendo sido priorizados o risco de queda e a crise de saúde aguda, dando seguimento aquelas que são preocupações já constantes na literatura na aplicação do TCM com vista à diminuição dos internamentos hospitalares. No entanto e tendo em conta a polimedicação acima 
referenciada, este é também um diagnóstico que deve ser relevado na perspectiva de um efetivo trabalho de equipe em estreita colaboração com a equipa médica e, eventualmente, nutricionista, caso se trate de suplementos alimentares substituíveis por uma alimentação adequada.

\section{Conclusões:}

A aplicação deste modelo em Portugal consubstancia-se numa mudança de paradigma, na medida em que a intervenção se foca no nível primário e terciário e com um acompanhamento precoce do utente, acompanhamento este que inicia e termina no domicílio, não obstante o internamento ou reinternamento que possa advir ao longo do tempo. É assim corroborada a pertinência da aplicação deste modelo pela elevada fragilidade do idoso em contexto domiciliário e a necessidade de um acompanhamento especializado. Numa próxima fase será expectável a validação da existência ou não de internamentos bem como se existiu uma maior adesão ao regime terapêutico medicamentoso, a adesão a medidas de segurança no que respeita ao risco de queda e/ou à melhor gestão da doença crônica. A realização deste artigo permitiu o desenvolvimento de competências relacionadas com a pesquisa de literatura relevante, a reflexão crítica para aplicação na prática, bem como a utilização de instrumentos que permitirão um adequado diagnóstico de situação. A implementação de projetos neste âmbito permitirá a eliminação de barreiras no serviço nacional de saúde, bem como no restante sistema, possibilitando o acompanhamento do idoso no seu contexto, reduzindo os internamentos, bem como todos os seus custos associados e aumentando a eficiência do acompanhamento do utente. Estamos perante um novo paradigma de gestão na saúde, com possibilidade de ganhos efetivos e um aproximar de profissionais e utentes entre os vários níveis de cuidados. 


\section{Referências}

1. Despacho $\mathrm{n}^{\mathrm{o}}$ 1400-A/2015. Aprova o Plano Nacional para a Segurança dos Doentes 20152020 [Portugal]. Diário da República [Internet]. 2015;28(2):4-9. Available from: https://dre.pt/application/file/664 57154

2. Sousa-Pinto B, Gomes AR, Oliveira A, Ivo C, Costa G, Ramos J, et al. Reinternamentos hospitalares em Portugal na última década. Acta Med Port. 2013;26(6):711-20.

3. Occelli P, Touzet S, Rabilloud M, Ganne C, Poupon Bourdy S, Galamand B, et al. Impact of a transition nurse program on the prevention of thirty-day hospital readmissions of elderly patients discharged from short-stay units: study protocol of the PROUST stepped-wedge cluster randomised trial. BMC Geriatr [Internet]. 2016;16(1):57. Available from: https://doi.org/10.1186/s12877016-0233-2

4. Boyle J, Speroff T, Worley K, Cao A, Goggins K, Dittus RS, et al. Low Health Literacy Is Associated with Increased
Transitional Care Needs in Hospitalized Patients. J Hosp Med [Internet]. 2017 Nov;12(11):91824. Available from: http://search.ebscohost.com/login .aspx?direct=true $\& \mathrm{db}=\mathrm{mdc} \& \mathrm{AN}$ $=29091980 \&$ lang $=$ ptbr\&site=ehost-live

5. Direção-Geral da Saúde. Programa Nacional para a Saúde das Pessoas Idosas. 2006.

6. Zimerman G. Velhice - Aspetos Biopsicossociais. Artmed Editora; 2000.

7. EUROSTAT. Estatísticas do emprego - Statistics Explained [Internet]. 2017 [cited 2019 Mar 25]. Available from: https://ec.europa.eu/eurostat/stati stics-

explained/index.php?title=Emplo yment_statistics/pt

8. Kahlon S, Pederson J, Majumdar SR, Belga S, Lau D, Fradette M, et al. Association between frailty and 30-day outcomes after discharge from hospital. Can Med Assoc J [Internet]. 2015 Aug 11;187(11):799 LP $\quad-804$. Available from: http://www.cmaj.ca/content/187/ 11/799.abstract

9. Andrade A do N, Fernandes M das GM, Nóbrega MML da, 
Garcia TR, Costa KN de FM. Análise do conceito fragilidade em idosos. Texto Context. 2012;

10. Meleis AI, Sawyer LM, Im E-O, Messias DKH, Schumacher K. Experiencing transitions: an emerging middle-range theory. Adv Nurs Sci. 2000;23(1):12-28.

11. World Health Organization. Hand Hygiene in Health Care First Global Patient Safety Challenge Clean Care is Safer Care. World Heal Organ [Internet]. 2017;30(1):64. Available from: http://whqlibdoc.who.int/publicat ions/2009/9789241597906_eng.p df

12. Finlayson K, Chang AM, Courtney MD, Edwards HE, Parker AW, Hamilton $\mathrm{K}$, et al. Transitional care interventions reduce unplanned hospital readmissions in high- risk older adults. BMC Health Serv Res. 2018;9:1-10.

13. Devriendt E, Heeren P, Fieuws S, Wellens NIH, Deschodt M, Flamaing $\mathrm{J}$, et al. Unplanned Readmission prevention by Geriatric Emergency Network for Transitional care ( URGENT ): protocol of a prospective single centre quasi- experimental study. BMC Geriatr. 2018;18:1-11.
14. Soto GE, Huenefeldt EA, Hengst MN, Reimer AJ, Samuel SK, Samuel SK, et al. Implementation and impact analysis of a transitional care pathway for patients presenting to the emergency department with cardiac-related complaints. BMC Health Serv Res. 2018;18:1-11.

15. Fabrício-Wehbe SCC, Schiaveto FV, Vendrusculo TRP, Haas VJ, Dantas RAS, Rodrigues RAP. Cross-cultural adaptation and validity of the "Edmonton Frail Scale - EFS" in a Brazilian elderly sample . Vol. 17, Revista LatinoAmericana de Enfermagem . scielo ; 2009. p. 1043-9.

16. Martins D, Carvalho I, Cordeiro N, Pinheira V. Contributo para a validação da Edmont Frail Scale (EFS). In: $8^{\circ}$ Congresso Internacional Luso-Espanhol sobre Envelhecimento Positivo e Solidariedade Intergeracional. 2012.

17. Costa-Dias MJM da, Ferreira PL, Oliveira AS. Adaptação cultural e linguística e validação da Escala de Quedas de Morse . Vol. serIV, Revista de Enfermagem Referência . scielopt ; 2014. p. 717.

18. Amaro F. Escala de Graffar 
adaptada. AM B Costa, FR Leitão,

J Santos, JV Pinto, MN Fino,

Currículos funcionais. 1996;2.

\section{Participação dos autores:}

Nascimento TFR trabalhou na concepção teórica, coleta de dados, análise estatística e elaboração e redação final do texto;

Costa MLGPV trabalhou na concepção teórica, elaboração e redação final do texto;

Recebido: 30.11.2019

Revisado: 09.08.2019

Aprovado: 16.01.2019 